\title{
Innovación docente en planificación hidrológica
}

\section{Innovative teaching in water resource planning}

ISABel Clara Rodríguez Medina

ORCID: https://orcid.org/0000-0001-7452-1552

Universidad de Sevilla

Departamento de Ingeniería

Aeroespacial y Mecánica de Fluidos

irodriguez8@us.es

Fecha de recepción: 22/11/2019

Fecha de aceptación: 26/11/2019

DOI: http://dx.doi.org/10.12795/9788447221912.114

Pp.: 2529-2549 


\section{Resumen}

Se ha implantado un ciclo de mejora durante el curso 2019/20 en la asignatura "Planificación Hidrológica" de segundo curso del Master de Ingeniería de Caminos, Canales y Puertos de la Universidad de Sevilla. Se describe el modelo metodológico, el mapa de contenidos y la relación detallada de actividades. La parte práctica consta de 10 prácticas a realizar en los talleres conceptuales. En ellas se hace uso del software ArcView, Aquatool, así como la utilización de varias páginas webs de organismos oficiales y bases de datos públicas. Para dinamizar las clases se introducen prácticas con la aplicación Kahoot así cómo la visualización de videos de Youtube enviados mediante la aplicación de mensajería instantánea de Whatsapp. Se ha utilizado un contrato colaborativo de trabajo para fomentar el trabajo en equipo dando forma al grupo de consulting de ingeniería. Los resultados obtenidos tanto en los objetivos alcanzados de los alumnos cómo en la evaluación del profesor son muy satisfactorios.

Palabras clave: Planificación Hidrológica, Master de Ingeniería de Caminos, Canales y Puertos, Docencia Universitaria, Experimentación docente Universitaria.

\section{Abstract}

Some improvements have been implemented during the academic year 2019/20 in the subject "Water Resource Planning" of the second year of the Master Degree in Road, Channel and Port Engineering of the University of Seville. The methodological model, the content map and a detailed activity list are described. The practical part consists of 10 practices to do in conceptual workshops. ArcView software, Aquatool, as well as some official institution websites and some public databases will be used. Some practices using the Kahoot app will be used in order to activate the courses, as well as some YouTube videos sent by Whatsapp. A collaboration agreement has been used to promote team working. The results obtained in the goals achieved by the students and the lecturer evaluation, are very satisfactory.

Keywords: Water Resource planning, Master Degree in Road, Channel and Port Engineering, University lectures, University lecture experimentation. 


\section{Contexto de la intervención}

El ciclo de mejora propuesto se va a desarrollar en la asignatura "Planificación Hidrológica", enseñanza que se imparte en la Escuela Técnica Superior de Ingenieros de la Universidad de Sevilla dentro del Master de Ingeniería de Caminos, Canales y Puertos. La asignatura está organizada en 5 bloques temáticos todos ellos con un carácter teórico-práctico.

La Escuela de Ingenieros dispone de todos los medios necesarios para el desarrollo de la docencia habitual. Parte de las clases se imparten en el aula habitual con ordenador, proyector y pizarra, y la otra parte de las clases se imparte en el Centro de Cálculo de la Escuela donde se dispone del software necesario.

El número de alumnos matriculados en el curso 2019/2020 es 19. Los alumnos matriculados son alumnos que se encuentran en su último año de formación académica y dominan muchos conceptos teóricos y prácticos con un alto grado de conocimiento en numerosas herramientas informáticas de cálculo y representación gráfica.

\section{Diseño previo del Ciclo de Mejora en el Aula (CIMA)}

El ciclo de mejora implantado en el curso 2019-2020 en la asignatura Planificación Hidrológica tiene como objetivo avanzar en el conocimiento real y llevarlo a la práctica en la realización de un plan hidrológico de cuenca. Para ello se implantan varios talleres conceptuales llevando al alumno a realizar la simulación de un sistema de explotación de recursos hídricos mediante herramientas y software específico dentro del marco legal existente. Se orienta la asignatura de forma que los alumnos adquieran todos los conocimientos necesarios tanto legislativos, teóricos e informáticos para poder elaborar un plan hidrológico de Demarcación. 


\section{Conexión con el proceso previo}

Esta experimentación docente se basa en una experiencia anterior con la que el ciclo de mejora en el aula (CIMA) que he diseñado tiene conexión y continuidad. Se trata del Segundo CIMA que realicé cómo experiencia en el desarrollo del Curso General de Experiencia Universitaria en la asignatura de Hidrología de segundo curso de la titulación de Grado en Ingeniería Civil (GIC) que se impartió en la Escuela Técnica Superior de Ingenieros de la Universidad de Sevilla, durante el segundo cuatrimestre del curso académico 2017/2018. De dicha experiencia docente envié comunicación a las V Jornadas de Formación e Innovación Docente del Profesorado de la Universidad de Sevilla. (Rodriguez, 2018).

Dicho CIMA tuvo una duración de 8 horas y se realizó el estudio de un Cuenca Hidrológica Ontime-Online. En general el resultado del CIMA fue satisfactorio ya que se consiguieron los objetivos.

Las actividades se desarrollaron en el tiempo que estaba previsto y se destaca el interés de los alumnos y participación de los alumnos que fue muy buena. La evaluación del aprendizaje de los alumnos se realizó mediante un cuestionario inicial y final a partir del cual se elaboraron las escaleras de aprendizaje de cada uno de los alumnos. Mi experiencia personal fue muy positiva suponiendo para mí un punto de inflexión en mi ejercicio docente. Este buen resultado me planteó como objetivo realizar esta experiencia en la asignatura de Planificación Hidrológica del segundo curso del Master de Ingenieros de Caminos, Canales y Puertos.

Las principales conclusiones que se obtuvieron fueron: 
Cuestiones a mantener:

a) Reconfigurar la metodología de la enseñanza: Realizar talleres conceptuales

b) Poner a los estudiantes a trabajar de manera continuada y haciéndolo que sean protagonistas en primera persona de la enseñanza

c) Conocer y analizar los modelos mentales de los estudiantes mediante cuestionarios y realizar una evaluación continua de los conocimientos por medio de la entrega de trabajos y exámenes por bloques de aprendizaje

Cambios a introducir en un futuro:

a) Plantear un modelo colaborativo mediante un contrato de trabajo

b) Enviar un mail previo a cada una de las clases anteponiendo lo que se va a realizar

c) Introducir nuevas tecnologías cómo Kahoot, videos a ver en el móvil con auriculares

d) Introducir un diario y un glosario a realizar de forma colaborativa con los alumnos en cada clase

e) Introducir la evaluación del docente y del diseño didáctico durante el proceso mediante un kahoot anónimo del profesor y un formulario personalizado de ciertas cuestiones

A partir de la experiencia anterior se procedió al diseño del presente CIMA caracterizado por:

- Mapa de Contenidos o Problemas

- Mantener en el CIMA el mismo modelo metodológico utilizado anteriormente

- Diseñar una secuencia de actividades para la asignatura completa

- Para el seguimiento de la evolución de los estudiantes a lo largo del CIMA se realizarán los cuestionarios inicial y final para cada uno de los bloques y se elaborarán las Escaleras de Aprendizaje 
- Para la evaluación del docente y del diseño didáctico del CIMA se realizará un kahoot anónimo, un diario del profesor y un formulario personalizado

El Ciclo de Mejora propuesto para la asignatura completa tiene una duración de 48 horas. Al tener asignada la asignatura 4 horas semanales, tendrá una duración de 4 meses para su desarrollo, de octubre a enero. Las sesiones tienen una duración de 1 hora 50 minutos cada una de ellas y se impartirán tanto en el aula cómo en el Centro de Cálculo desarrollado en ambas los correspondientes talleres conceptuales. Gran parte de la asignatura tiene un carácter eminentemente práctico por lo que se ha diseñado una secuencia de actividades a desarrollar a lo largo del curso de tal forma que cada alumno utiliza un ordenador con sofware específico, móvil y auriculares con el que se realizan diversas actividades en varias sesiones. La evaluación final de cada alumno se realiza por medio de una evaluación continua en la que se puntúa porcentualmente los trabajos entregados en los talleres así como un examen.

Debido a las exigencias del calendario y al tener que entregar los resultados de esta experiencia antes del 15 de Noviembre, se presentan los resultados obtenidos en el CIMA hasta esta fecha.

\section{Modelo metodológico}

El modelo metodológico posible diseñado es el basado en la reelaboración de ideas de los alumnos a partir del planteamiento de un problema, es decir, se abre la clase con el planteamiento de un problema seguido de la exposición de ideas de los alumnos, después se realizan actividades de contraste y por último se extraen las conclusiones. A continuación se representa un esquema con el modelo metodológico que se propone: 
Modelo metodológico aplicado:

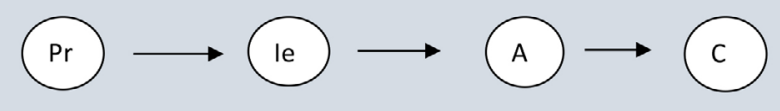

Figura 1. Modelo metodológico aplicado

El Ciclo de Mejora propuesto para impartir en la asignatura de Planificación Hidrológica se está desarrollando en 26 sesiones. La metodología de trabajo se ha diseñado con sesiones y actividades pensadas y programadas haciendo que los alumnos "sean protagonistas" en primera persona de su aprendizaje, interactuando con el profesor y trabajando "on-line" y "on-time". De esta manera se pretende crear un clima de trabajo enriquecedor en el que se pretende ilusionar al alumno con el trabajo que tiene que desarrollar, así como con la forma de aprender y trabajar.

Jornadas de Formación e Innovación Docente del Profesorado | № 2 (2019) Esta obra se distribuye con la licencia Creative Commons 
El Mapa de Contenidos que se presenta es el de la asignatura completa.

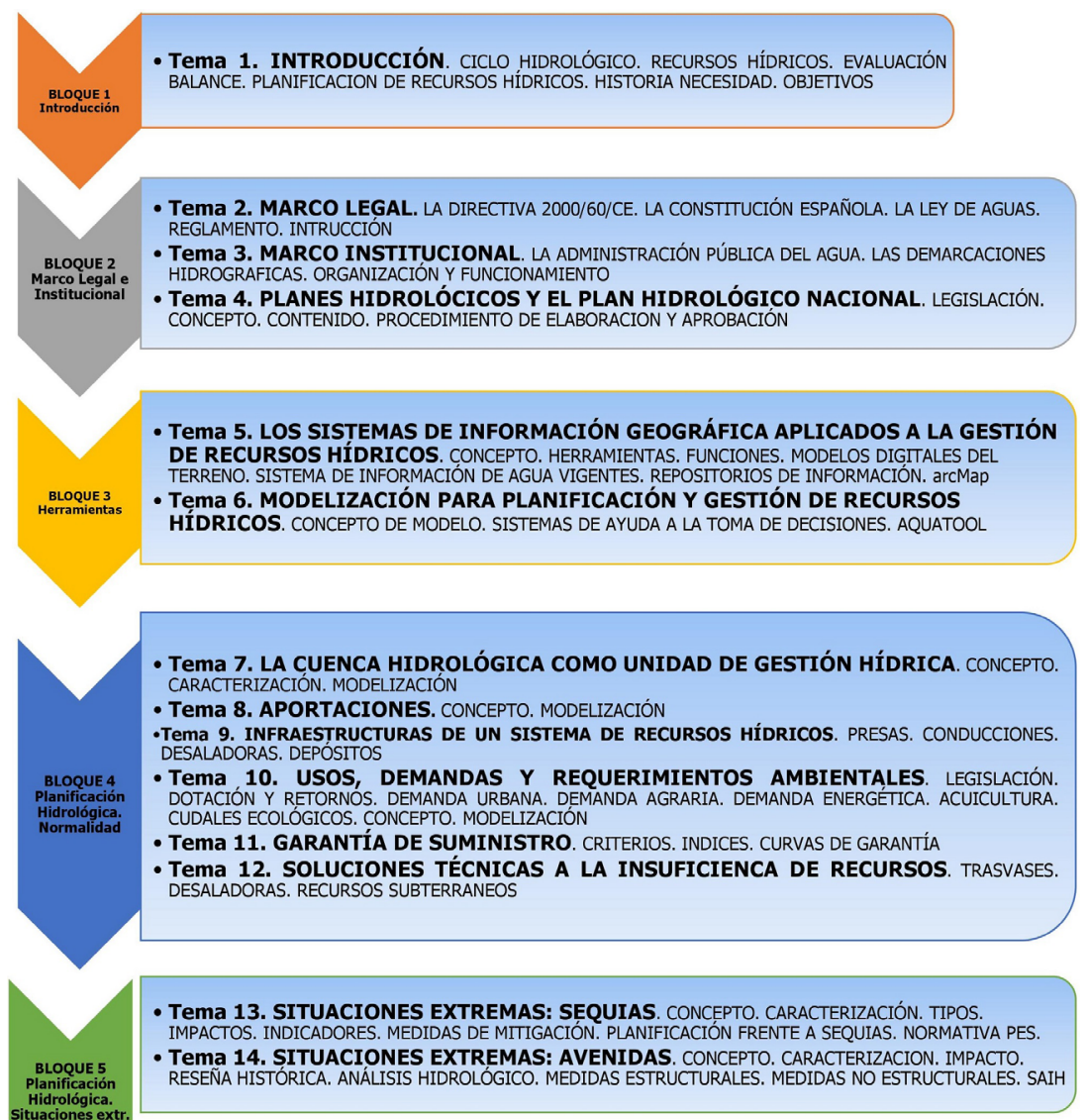

Figura 2. Mapa de contendidos

\section{Secuencia de actividades}

Paralelamente a la realización del diseño de este mapa de contenidos se ha desarrollado una secuencia temporalizada de actividades para cada una de las sesiones.

Jornadas de Formación e Innovación Docente del Profesorado | № 2 (2019) Esta obra se distribuye con la licencia Creative Commons Reconocimiento-NoComercial-SinObraDerivada Internacional (CC BY-NC-ND 4.0.) 
Tabla 1. Secuencia de actividades. Sesión 1

\begin{tabular}{|c|c|c|}
\hline № Sesión: & \multicolumn{2}{|l|}{1} \\
\hline Nombre: & \multicolumn{2}{|c|}{ Presentación de la asignatura y Tema 1: Introducción } \\
\hline Fecha: & \multicolumn{2}{|c|}{25 de Octubre de 2019} \\
\hline Tiempo: & \multicolumn{2}{|c|}{1 hora y 50 min } \\
\hline $\begin{array}{l}\text { Lugar de } \\
\text { impartición: }\end{array}$ & \multicolumn{2}{|l|}{ Aula } \\
\hline Actividad & $\begin{array}{l}\text { Tiempo } \\
(\mathrm{min})\end{array}$ & Descripción \\
\hline \multicolumn{3}{|c|}{ Antes de la clase se envía un mail a cada alumno presentando la clase } \\
\hline 1.1 & 5 & Saludo. \\
\hline 1.2 & 30 & $\begin{array}{l}\text { Presentación de la asignatura. Objetivos. Temario. } \\
\text { Metodología de enseñanza. Presentación de las } 10 \\
\text { prácticas a realizar a lo largo del curso. Presentación } \\
\text { de los sistemas de evaluación y calificación. }\end{array}$ \\
\hline 1.3 & 10 & $\begin{array}{l}\text { Presentación y lectura del contrato colaborativo de } \\
\text { trabajo. }\end{array}$ \\
\hline 1.4 & 5 & Presentación de la bibliografia \\
\hline 1.5 & 30 & $\begin{array}{l}\text { Exposición del Tema 1- Introducción mediante } \\
\text { diapositivas. }\end{array}$ \\
\hline 1.6 & 20 & $\begin{array}{l}\text { Se les deja un artículo en enseñanza virtual para } \\
\text { leer y reflexionar y resumir las ideas principales }\end{array}$ \\
\hline 1.7 & 10 & $\begin{array}{l}\text { Bombardeo de ideas del artículo anterior y sirven } \\
\text { cómo conclusión }\end{array}$ \\
\hline Total & 110 & \\
\hline
\end{tabular}

Tabla 2. Secuencia de actividades. Sesión 2

\begin{tabular}{|c|c|c|}
\hline № Sesión: & \multicolumn{2}{|l|}{2} \\
\hline Nombre: & \multicolumn{2}{|c|}{ Tema 2- Marco legal } \\
\hline Fecha: & \multicolumn{2}{|c|}{25 de Octubre de 2019} \\
\hline Tiempo: & \multicolumn{2}{|c|}{1 hora y $50 \mathrm{~min}$} \\
\hline $\begin{array}{l}\text { Lugar de } \\
\text { impartición: }\end{array}$ & \multicolumn{2}{|l|}{ Aula } \\
\hline Actividad & $\begin{array}{l}\text { Tiempo } \\
\text { (min) }\end{array}$ & Descripción \\
\hline \multicolumn{3}{|c|}{$\begin{array}{l}\text { Antes de la clase se envía un mail a cada alumno presentando la clase y los } \\
\text { objetivos a alcanzar. }\end{array}$} \\
\hline 2.1 & 10 & Saludo. Introducción. \\
\hline 2.2 & 5 & Recogida de contrato colaborativo \\
\hline
\end{tabular}




\begin{tabular}{|l|l|l|}
\hline 2.3 & 10 & Cuestionario inicial bloque 1 \\
\hline 2.4 & 10 & $\begin{array}{l}\text { Se les presenta en Enseñanza Virtual la } \\
\text { documentación a utilizar en la clase: Legislación } \\
\text { vigente y T1 }\end{array}$ \\
\hline 2.5 & 50 & $\begin{array}{l}\text { Exposición del Tema 2-Marco legal, mediante } \\
\text { diapositivas a la vez que se van realizando las } \\
\text { diversas cuestiones de la T1 }\end{array}$ \\
\hline 2.6 & 10 & Entrega de la práctica \\
\hline 2.7 & 5 & Lectura del diario del día por un alumno \\
\hline 2.8 & 10 & Elaboración del glosario por un alumno \\
\hline Total & 110 & \\
\hline
\end{tabular}

Tabla 3. Secuencia de Actividades. Sesión 3

\begin{tabular}{|l|l|l|}
\hline № Sesión: & 3 \\
\hline Nombre: & $\begin{array}{l}\text { Tema 3- Marco institucional. Las Demarcaciones Hidrográficas en } \\
\text { España. }\end{array}$ \\
\hline Fecha: & \multicolumn{3}{|l|}{ 30 de Octubre de 2019 } \\
\hline Tiempo: & 1 hora y 50 min \\
\hline $\begin{array}{l}\text { Lugar de } \\
\text { impartición: }\end{array}$ & Aula & \\
\hline Actividad & $\begin{array}{l}\text { Tiempo } \\
\text { (min) }\end{array}$ & Descripción \\
\hline $\begin{array}{l}\text { Antes de la clase se envía un mail a cada alumno presentando la clase y los } \\
\text { objetivos a alcanzar. }\end{array}$ & 10 & Saludo. Introducción. \\
\hline 3.1 & 5 & $\begin{array}{l}\text { Se les presenta en Enseñanza Virtual la } \\
\text { documentación a utilizar en la clase }\end{array}$ \\
\hline 3.2 & 60 & $\begin{array}{l}\text { Exposición del Tema 3-Marco Territorial mediante } \\
\text { diapositivas a la vez que se van realizando las } \\
\text { diversas cuestiones de la T2 }\end{array}$ \\
\hline 3.3 & 10 & $\begin{array}{l}\text { Vemos video en youtube sobre el tema en los } \\
\text { móviles de los alumnos y auriculares }\end{array}$ \\
\hline 3.4 & 10 & Entrega de la práctica \\
\hline 3.5 & 5 & Lectura del diario del día por un alumno \\
\hline 3.6 & 10 & Elaboración del glosario por un alumno \\
\hline 3.7 & 10 & \\
\hline Total & &
\end{tabular}

Jornadas de Formación e Innovación Docente del Profesorado | № 2 (2019)

Esta obra se distribuye con la licencia Creative Commons

Reconocimiento-NoComercial-SinObraDerivada

Internacional (CC BY-NC-ND 4.0.) 
Tabla 4. Secuencia de Actividades. Sesión 4

\begin{tabular}{|c|c|c|}
\hline № Sesión: & \multicolumn{2}{|l|}{4} \\
\hline Nombre: & \multicolumn{2}{|c|}{$\begin{array}{l}\text { Tema } 5 \text { - Los Sistemas de Información Geográfica aplicados a la } \\
\text { gestión de recursos hídricos. }\end{array}$} \\
\hline Fecha: & \multicolumn{2}{|c|}{6 de Noviembre } \\
\hline Tiempo: & $\begin{array}{l}1 \text { hora y } 50 \\
\min \end{array}$ & \\
\hline $\begin{array}{l}\text { Lugar de } \\
\text { impartición: }\end{array}$ & $\begin{array}{l}\text { Centro de } \\
\text { Cálculo }\end{array}$ & \\
\hline Actividad & $\begin{array}{l}\text { Tiempo } \\
(\min )\end{array}$ & Descripción \\
\hline \multicolumn{3}{|c|}{$\begin{array}{l}\text { Antes de la clase se envía un mail a cada alumno presentando la clase y los } \\
\text { objetivos a alcanzar. }\end{array}$} \\
\hline 4.1 & 4 & Saludo. Introducción. Presentación. \\
\hline 4.2 & 15 & Cuestionario inicial Bloque temático 3 \\
\hline 4.3 & 30 & $\begin{array}{l}\text { Exposición del Tema 5- Los sistemas de Información } \\
\text { Geográfica aplicados a la gestión de recursos } \\
\text { hídricos mediante diapositivas }\end{array}$ \\
\hline 4.4 & 45 & $\begin{array}{l}\text { Clase online de ArcGis realizando diversos ejercicios } \\
\text { prácticos }\end{array}$ \\
\hline 4.5 & 10 & $\begin{array}{l}\text { Presentación de diversos repositorios oficiales de } \\
\text { fuentes y bases de datos }\end{array}$ \\
\hline 4.6 & 3 & Lectura del diario del día por un alumno \\
\hline 4.7 & 3 & Elaboración del glosario por un alumno \\
\hline Total & 110 & \\
\hline
\end{tabular}

Tabla 5. Secuencia de Actividades. Sesión 5

\begin{tabular}{|c|c|c|}
\hline № Sesión: & \multicolumn{2}{|l|}{5} \\
\hline Nombre: & \multicolumn{2}{|c|}{ Clase en el Centro de Cálculo. Taller conceptual. } \\
\hline Fecha: & \multicolumn{2}{|c|}{8 de Noviembre de 2019} \\
\hline Tiempo: & \multicolumn{2}{|c|}{1 hora y $50 \mathrm{~min}$} \\
\hline $\begin{array}{l}\text { Lugar de } \\
\text { impartición: }\end{array}$ & \multicolumn{2}{|c|}{ Centro de Cálculo } \\
\hline Actividad & Tiempo (min) & Descripción \\
\hline \multicolumn{3}{|c|}{$\begin{array}{l}\text { Antes de la clase se envía un mail a cada alumno presentando la clase y los } \\
\text { objetivos a alcanzar }\end{array}$} \\
\hline 5.1 & 5 & Saludo. Introducción. Presentación de la T3 \\
\hline
\end{tabular}

Jornadas de Formación e Innovación Docente del Profesorado | № 2 (2019)

Esta obra se distribuye con la licencia Creative Commons

Reconocimiento-NoComercial-SinObraDerivada

Internacional (CC BY-NC-ND 4.0.) 


\begin{tabular}{|l|l|l|}
\hline 5.2 & 5 & $\begin{array}{l}\text { Se les presenta en Enseñanza Virtual la } \\
\text { documentación a utilizar en la clase y las } \\
\text { herramientas }\end{array}$ \\
\hline 5.3 & 100 & Desarrollo de la T3 \\
\hline Total & 110 & \\
\hline
\end{tabular}

Tabla 6. Secuencia de Actividades. Sesión 6

\begin{tabular}{|c|c|c|}
\hline № Sesión: & \multicolumn{2}{|l|}{6} \\
\hline Nombre: & \multicolumn{2}{|c|}{$\begin{array}{l}\text { Tema 7- la cuenca hidrológica cómo unidad de gestión. Taller } \\
\text { conceptual. }\end{array}$} \\
\hline Fecha: & \multicolumn{2}{|c|}{13 de Noviembre de 2019} \\
\hline Tiempo: & \multicolumn{2}{|c|}{1 hora y $50 \mathrm{~min}$} \\
\hline $\begin{array}{l}\text { Lugar de } \\
\text { impartición: }\end{array}$ & \multicolumn{2}{|c|}{ Centro de Cálculo } \\
\hline Actividad & Tiempo (min) & Descripción \\
\hline \multicolumn{3}{|c|}{$\begin{array}{l}\text { Antes de la clase se envía un mail a cada alumno presentando la clase y los } \\
\text { objetivos a alcanzar }\end{array}$} \\
\hline 6.1 & 5 & $\begin{array}{l}\text { Saludo. Introducción. Presentación de la T4 a } \\
\text { entregar al final de la clase on-line }\end{array}$ \\
\hline 6.2 & 10 & $\begin{array}{l}\text { Se les presenta en Enseñanza Virtual la } \\
\text { documentación a utilizar en la clase y las } \\
\text { herramientas }\end{array}$ \\
\hline 6.3 & 90 & $\begin{array}{l}\text { Exposición del Tema 7- La cuenca hidrológica cómo } \\
\text { unidad de gestión, mediante diapositivas a la vez } \\
\text { que se van realizando las diversas cuestiones de } \\
\text { la T4 }\end{array}$ \\
\hline 6.4 & 5 & Entrega de la T3 \\
\hline Total & 110 & \\
\hline
\end{tabular}

Tabla 7. Secuencia de Actividades. Sesión 7

\begin{tabular}{|c|c|c|}
\hline № Sesión: & \multicolumn{2}{|l|}{7} \\
\hline Nombre: & \multicolumn{2}{|c|}{ Evaluación del alumno. Evaluación del profesor. } \\
\hline Fecha: & \multicolumn{2}{|c|}{15 de Noviembre de 2019} \\
\hline Tiempo: & \multicolumn{2}{|l|}{1 hora y $50 \mathrm{~min}$} \\
\hline $\begin{array}{l}\text { Lugar de } \\
\text { impartición: }\end{array}$ & \multicolumn{2}{|c|}{ Centro de Cálculo } \\
\hline Actividad & Tiempo (min) & Descripción \\
\hline \multicolumn{3}{|c|}{$\begin{array}{l}\text { Antes de la clase se envía un mail a cada alumno presentando la clase y los } \\
\text { objetivos a alcanzar }\end{array}$} \\
\hline
\end{tabular}

Jornadas de Formación e Innovación Docente del Profesorado | № 2 (2019)

Esta obra se distribuye con la licencia Creative Commons

Reconocimiento-NoComercial-SinObraDerivada

Internacional (CC BY-NC-ND 4.0.) 


\begin{tabular}{|l|l|l|}
\hline 7.1 & 5 & Saludo. Introducción. Presentación. \\
\hline 7.2 & 30 & $\begin{array}{l}\text { Se realiza examen de los contenidos teóricos } \\
\text { impartidos. }\end{array}$ \\
\hline 7.3 & 60 & $\begin{array}{l}\text { Se realiza examen del Tema de Herramientas. Tema } \\
5 .\end{array}$ \\
\hline 7.4 & 15 & $\begin{array}{l}\text { Se entrega un cuestionario sobre sobre otras } \\
\text { cuestiones }\end{array}$ \\
\hline Total & 110 & \\
\hline
\end{tabular}

A lo largo del curso se realizan 10 talleres conceptuales que tienen como resultado la elaboración de 10 prácticas. Cada uno de estos talleres están centrados en alcanzar los principales objetivos docentes de la asignatura, ya sean aptitudinales, conceptuales o procedimentales, por los que su realización tiene el doble objetivo de "aprender en clase" y ser "evaluados".

La realización del cuestionario inicial nos proporciona el nivel de conocimientos de los alumnos y nos permite observar su evolución y sobre todo identificar los posibles objetivos no alcanzados de forma genérica en los alumnos para identificar puntos de mejora en la docencia para futuros cursos.

\section{Aplicación del CIMA}

Las actividades relacionadas anteriormente se llevaron a cabo entre los meses de octubre y noviembre de 2019 con algunas modificaciones en calendario debido a causas como:

- Corte de de clases debida a la Jornadas BIM en la Ingeniería

- Modificación de fechas de clase a petición de los alumnos

- No disponibilidad de licencias de ArcView

Las causas expuestas anteriormente hicieron que se retrasara una semana su impartición; realizando la sesión número 7 de evaluación prevista para el día 15 de 
noviembre el día 22 de noviembre y no pudiendo impartir el Tema 7 antes de la evaluación realizada.

Las prácticas se realizaron con el Software ArcView y realizando unos cuestionarios pensados para fijar los conceptos principales vistos en clase. Todo ello lleva a los alumnos a poder realizar un "Estudio de Planificación Hidrológica" de un Sistema de Explotación de Recursos completo.

El estudiante no está acostumbrado a trabajar con software de GIS y a realizar simulaciones de sistemas de Explotación de Recursos Hídricos. La docencia tradicional habría consistido en teoría y problemas impartidos mediante clases magistrales (García-Pérez, 2000). La innovación fundamental introducida es constituir un grupo de trabajo colaborativo en el que los estudiantes trabajan compartiendo información, contrastando resultados simulando ser un consulting de ingeniería "Solagua" acercando de esta manera al estudiante a la realidad y trabajando en la realización de estos estudios de planificación hidrológica usando el software más idóneo disponible en el mercado así cómo bases de datos y servidores públicos actuales. (Delval, 2000), (Alba \& Porlán, 2017).

Las actividades diseñadas se han desarrollado tanto a nivel individual cómo en grupos, de manera que los alumnos han interactuado entre ellos y aprendido/corregido unos de otros (López, 2005; MaríYtarte et al., 2016).

La innovación docente y la "capacidad de sorpresa" a los alumnos (los alumnos no esperan una clase con simulaciones, actividades ontime online, WhastsApp y kahoot) motiva a los mismos y les mantiene la atención durante la práctica docente (Bain, 2007; García-Pérez, 2000; Roth et al., 2007; Miguel-Dávila et al., 2012), lo que unido a la obligación de entregar las prácticas en un corto espacio de tiempo y la realización de exámenes de forma continua, les hace mantener la atención en clase e ir aprendiendo de forma continua. 


\section{Evaluación del CIMA puesto en práctica}

\section{Evaluación de los alumnos}

Para evaluar el aprendizaje de los alumnos en cada tema se han comparado sus conocimientos iniciales y finales. Para conocer los contenidos iniciales se preparó un cuestionario inicial a desarrollar el primer día de inicio de cada bloque en el que se analizan los conocimientos que tienen los alumnos. La evaluación del conocimiento aprendido por los alumnos se ha realizado por medio de la evaluación de cada una de las prácticas entregadas y por la realización de un examen teórico-práctico.

\section{Tabla 8. Cuestionario inicial}

\begin{tabular}{|l|}
\hline ¿Qué es una cuenca hidrológica? \\
\hline ¿Qué es una Demarcación Hidrográfica? \\
\hline ¿Qué legislación conoces donde se definan los conceptos anteriores? \\
\hline ¿Qué es un Sistema de Información Geográfica? \\
\hline $\begin{array}{l}\text { ¿Has trabajado o utilizado algún GIS anteriormente de tal manera que consideres } \\
\text { que tienes un nivel inicial o medio? Describe lo que puedes hacer. }\end{array}$ \\
\hline
\end{tabular}

Tabla 9. Cuestionario final

\begin{tabular}{|l|}
\hline ¿Qué es una cuenca hidrológica? \\
\hline ¿Qué es una Demarcación Hidrográfica? \\
\hline ¿Qué legislación conoces en la que se definan? \\
\hline ¿Qué es un Sistema de Información Geográfica? \\
\hline $\begin{array}{l}\text { ¿Has trabajado o utilizado algún GIS anteriormente de tal manera que consideres } \\
\text { que tienes un nivel inicial o medio? Describe lo que puedes hacer. }\end{array}$ \\
\hline
\end{tabular}

Se establecen 5 niveles de conocimiento para cada tarea: nulo, inicial, medio, avanzado y excelente. 
Los cuestionarios inicial y final no coinciden pero son suficientes como para evaluar los conocimientos de los alumnos y la evolución del aprendizaje en los contenidos conceptuales, procedimentales y aptitudinales.

De los 19 alumnos matriculados 15 han optado por el sistema de evaluación continua, lo que representa un porcentaje elevado. La asistencia a las sesiones no es obligatoria pero en todas ellas asistieron la práctica totalidad de los estudiantes.

Tal y como muestra la tabla 4, de los 15 alumnos que han optado por este sistema ningún alumno tuvo un nivel nulo, sólo 2 tuvieron un nivel bajo, 4 un nivel medio y 3 un nivel avanzado. Estos resultados se corresponden casi paralelamente con las pruebas escritas así cómo los alumnos que han asistido a clases en la totalidad o casi totalidad de las sesiones.

Tabla 10. Calificación de los estudiantes y evolución del aprendizaje

\begin{tabular}{|c|c|c|c|c|c|}
\hline Alumno & \multicolumn{3}{|c|}{ Prácticas } & \multicolumn{2}{c|}{ Examen Teoría } \\
\hline & T1 & T2 & T3 & Examen Teoría & Examen Práctica \\
\hline 1 & 7 & 7 & 7 & 6 & 8,00 \\
\hline 2 & 7 & 8 & 9 & 7 & 8,00 \\
\hline 3 & 7 & 7 & 7 & 5 & 5,00 \\
\hline 4 & 7 & 7 & 7 & 6 & 7,00 \\
\hline 5 & 8 & 8 & 10 & 9 & 10,00 \\
\hline 6 & 6 & 6 & 10 & 9 & 10,00 \\
\hline 7 & 5 & 5 & 5 & 6 & 6,00 \\
\hline 8 & 6 & 6 & 9 & 7 & 7,00 \\
\hline 9 & 5 & 5 & 5 & 6 & 8,00 \\
\hline 10 & 7 & 8 & 7 & 8 & 6,00 \\
\hline 11 & 7 & 7 & 7 & 7 & 9,00 \\
\hline 12 & 9 & 9 & 10 & 9 & 6,00 \\
\hline 13 & 8 & 8 & 8 & 2 & 2,00 \\
\hline 14 & 5 & 5 & 3 & 5 & 3,00 \\
\hline 15 & 5 & 5 & 4 & 5 & \\
\hline
\end{tabular}




\section{Evaluación del propio diseño y de mi intervención}

Para tener una idea del grado de satisfacción de los alumnos con el ciclo de mejora, se preparó un cuestionario anónimo en el que los alumnos valoraban con una puntuación de 1 a 5 varias cuestiones sobre contenido, metodología de trabajo y evaluación del profesor; además de un apartado donde podían escribir libremente sobre otros aspectos (siendo 1 totalmente en desacuerdo y 5 totalmente de acuerdo). Los resultados de la encuesta se muestran en la siguiente tabla:

Tabla 11. Valoración del ciclo de mejora por los estudiantes

\begin{tabular}{|l|c|}
\hline $\begin{array}{l}\text { Las herramientas utilizadas son las idóneas para realizar un análisis de } \\
\text { un sistema de explotación de recursos e recursos hídrico. }\end{array}$ & 4.7 \\
\hline La metodología de enseñanza me parece adecuada & 5 \\
\hline El software utilizado me será útil en mi vida profesional & 4 \\
\hline $\begin{array}{l}\text { Las clases en el Centro de Cálculo me han sido útiles para e interesantes } \\
\text { para aprender a utilizar las herramientas. }\end{array}$ & 4.2 \\
\hline Prefiero las clases tradicionales de teoría y problemas en pizarra. & 0 \\
\hline La intervención del profesor es adecuada & 4 \\
\hline $\begin{array}{l}\text { La forma de trabajar colaborativamente es enriquecedora y positiva para } \\
\text { nosotros. }\end{array}$ & 5 \\
\hline
\end{tabular}

Con sólo observar los resultados del cuestionario es evidente que el ciclo de mejora ha sido un éxito entre los alumnos, cumpliendo su objetivo en la mejora docente y motivación de los alumnos y que habrá que mantenerlo en cursos próximos.

La opinión de los alumnos refleja un alto grado de satisfacción en la metodología de la enseñanza, en la forma de trabajar colaborativamente, las practicas realizadas, los métodos de trabajo empleada y también con mi ejercicio docente.

Jornadas de Formación e Innovación Docente del Profesorado | № 2 (2019) Esta obra se distribuye con la licencia Creative Commons Reconocimiento-NoComercial-SinObraDerivada Internacional (CC BY-NC-ND 4.0.) 
Trabajar con en el modelo de enseñanza "dando clase con la boca cerrada" y el empleo de talleres conceptuales resulta exitoso y los resultados obtenidos y la forma en la que estos se alcanzan de una manera más amena y didáctica y por tanto enriquecedora resulta muy satisfactorio para los alumnos y para el docente.

La utilización del CIMA en la mejora de la práctica docente supone un nuevo éxito que avala esta forma de hacer docencia suponiendo una continuidad en las mejoras alcanzadas. La programación y temporización de las actividades, el buscarle un sentido a cada una de ellas, el realizar preguntas que despierten y mantengan la atención durante las clases, suponen un gran ejercicio de reflexión y preparación por parte del docente que se ve altamente recompensado con la satisfacción de saber que los alumnos aprenden de manera cómoda, amena, enriquecedora, haciéndolos pensar y trabajar y con un altísimo nivel de satisfacción por parte de ellos y consecuentemente con el del profesor.

El tratamiento de los datos y la representación de los resultados obtenidos mediante gráficos incita a la reflexión posterior de varias variables cómo pueden ser: el método empleado en el aprendizaje, el sistema de enseñanza, los objetivos alcanzados, etc. La elaboración de las escaleras y gráficos comparativos es interesante para analizar nuestra práctica docente y continuar con un proceso de mejora continua.

La aplicación del ciclo de mejora supone un punto de inflexión en mi manera de concebir la docencia aportando muchas cosas que abarcan desde la disposición de las aulas, la preparación de las clases, los talleres conceptuales, la forma de interactuar con los alumnos, de trabajar, de evaluar, de dar clase con la boca cerrada que más que un fin me parecen un principio de empezar a cambiar y seguir preparando Ciclos de Mejora al ser muy satisfactorio y enriquecedor en los sentidos comentados en párrafos anteriores. 
El Programa de Formación e Innovación Docente del Profesorado del año 2019 de la Universidad de Sevilla (FIDOP) me parece muy acertado y útil en su conjunto pues ofrece la posibilidad real de aprender a "hacer docencia" y de mejorar en nuestra profesión docente siendo una guía para afrontar mejor la práctica docente y pedagógica desde un modelo innovador de enseñanza. La realización del Ciclo de Mejora supone un inicio en el que se han sembrado las bases sólidas para empezar a andar un nuevo camino de mejora continua. De cara al futuro, la REFID supone un entorno seguro para realizar esta mejora continua de la práctica docente empleando nuevas técnicas innovadoras, recibiendo apoyo y guías de trabajo de otros compañeros más experimentados o no, pero que de una forma u otra se comparten muchas experiencias que enriquecen mucho y hacen que crezcamos todos como profesionales

El grado de satisfacción obtenido tanto en los conocimientos adquiridos por los alumnos cómo en la metodología de la enseñanza y el clima de trabajo en clase hacen que se mantengan los cambios introducidos para los próximos cursos en todas las sesiones teniendo en cuenta que hay que solucionar el problema de licencias para hacer uso del software ArcView. La solución consistirá en gestionar la adquisición de más licencias por parte del Departamento, Centro o Universidad de tal manera que estén disponibles en el Centro de Cálculo de la Escuela Técnica Superior de Ingeniería.

La incorporación en las sesiones de la entrega de la 10 Tareas a lo largo del curso y la obligación de la entrega de estas on-line al final de la clase, de un trabajo que va a ser evaluado, hacen que se mantenga la atención y la tensión durante el desarrollo de las clases asegurando que "no hay pérdida de tiempo" ni de atención por parte del alumno. 
La incorporación en las sesiones de elementos cómo la lectura de artículos y puesta en común, videos mediante la aplicación WhastsApp con auriculares y algunos kahoot para algunos temas pretenden aprovechar las nuevas tecnologías disponibles a día de hoy a la vez que introducen un elemento de entusiasmo y dinamismo a las clases. 


\section{Referencias bibliográficas}

Aguilar, M. (2009): Cómo animar a un grupo. Madrid. Editorial CCS.

Bain, K. (2007): Lo que hacen los mejores profesores universitarios. Valencia: Universidad de Valencia.

Cubero, R. (2005). Perspectivas constructivistas. La intersección entre el significado, la interacción y el discurso. Barcelona: Graó.

De Alba, N. y Porlān, R. (2017). La metodología de enseñanza. En R. Porlán (coord.), Enseñanza universitaria. Cómo mejorarla (pp. 37-54). Madrid: Morata.

Delval, J. (2000). Aprender en la vida y en la escuela. Madrid: Morata.

Finkel, D. (2008): Dar clase con la boca cerrada. Valencia: Publicaciones de la Universidad de Valencia.

García-Pérez, F. F. (2000): Los modelos didácticos como instrumento de análisis y de intervención en la realidad educativa. Biblio 3W. Revista Bibliográfica de Geografia y Ciencias Sociales, 207. En: http://www.ub.edu/geocrit/b3w-207.html

MaríYtarte, R. M., Moreno, R., Hipólito, N. (2016). Educación y ciudadanía. Propuestas educativas desde la controversia. Foro de Educación, 14(20), 49-69.

Miguel-Dávila, J. A., López-Berzosa, D., Martín-Sánchez, M. (2012). ¿Una participación activa del alumno pronostica una buena nota en el examen? (Does the active participation of students serve as a predictor of good marks achievement?). Working Papers on Operations Management, 3, 71-83.

Porlán, R. Coord. (2017). Enseñanzauniversitaria. Cómo mejorarla. Madrid: Morata.

Porlán, R. y MARTİN, J. (1991). El diario del profesor. Un recurso para la investigación en el aula. Sevilla: Díada.

RODRIGUEZ, I.C. (2018). La innovación docente en hidrología. Aplicación práctica. Monográfico Jornadas de Formación e Innovación Docente del Profesorado 1, pp. 655-670. 